\title{
HS-543 induces apoptosis of Imatinib-resistant chronic myelogenous leukemia with T315I mutation
}

\author{
Soo Jung Kim ${ }^{1, *}$, Kyung Hee Jung ${ }^{1, *}$, Hong Hua Yan ${ }^{1}$, Mi Kwon Son ${ }^{1}$, Zhenghuan \\ Fang ${ }^{1}$, Ye-Lim Ryu ${ }^{1}$, Hyunseung Lee ${ }^{1}$, Joo Han Lim ${ }^{1}$, Jun-Kyu Suh ${ }^{2}$, JinHee Kim ${ }^{3,4}$, \\ Soyoung Lee ${ }^{3,4}$, Sungwoo Hong ${ }^{3,4}$ and Soon-Sun Hong ${ }^{1}$ \\ ${ }^{1}$ College of Medicine, Inha University, Sinheung-dong, Jung-gu, Incheon, Republic of Korea \\ ${ }^{2}$ National Research Center for Sexual Medicine, College of Medicine, Inha University, Sinheung-dong, Jung-gu, Incheon, \\ Republic of Korea \\ ${ }^{3}$ Center for Catalytic Hydrocarbon Functionalizations, Institute for Basic Science (IBS), Republic of Korea \\ ${ }^{4}$ Department of Chemistry, Korea Advanced Institute of Science and Technology (KAIST), Daejeon, Republic of Korea \\ * These authors contributed equally to this work \\ Correspondence to: Soon-Sun Hong, email: hongs@inha.ac.kr \\ Sungwoo Hong, email: hongorg@kaist.ac.kr
}

Keywords: HS-543, Bcr-Abl, T315I, Chronic Myeloid Leukemia

Received: August 04, $2014 \quad$ Accepted: December 01,2014 Published: December 02, 2014

This is an open-access article distributed under the terms of the Creative Commons Attribution License, which permits unrestricted use, distribution, and reproduction in any medium, provided the original author and source are credited.

\section{ABSTRACT}

Chronic myeloid leukemia (CML) is characterized by a constitutive activation of Bcr-Abl tyrosine kinase. Bcr-Abl/T315I is the predominant mutation that causes resistance to Imatinib. In the present study, we synthesized a novel Bcr-Abl inhibitor, HS-543, and investigated its effect on cell survival or apoptosis in CML cells bearing Bcr-Abl/T315I (BaF3/T315I) or wild-type Bcr-Abl (BaF3/WT). HS-543 showed anti-proliferative effects in the BaF3/WT cells as well as the BaF3/T315I cells with resistance to Imatinib and strongly inhibited the Bcr-Abl signaling pathway in a dose-dependent manner. Furthermore, it significantly increased the sub $\mathbf{G}_{1}$ phase associated with early apoptosis, with increased levels of cleaved PARP and cleaved caspase-3, as well as the TUNEL-positive apoptotic cells. In addition, we found that HS-543 induced apoptosis with the loss of mitochondrial membrane potential by decreasing the expression of $\mathrm{Mcl-1}$ and survivin, together with increasing that of Bax. In BaF3/T315I xenograft models, HS-543 significantly delayed tumor growth, unlike Imatinib. Our results demonstrate that HS-543 exhibits the induction of apoptosis and anti-proliferative effect by blocking the Bcr-Abl signaling pathway in the T315Imutated Bcr-Abl cells with resistance to Imatinib. We suggest that HS-543 may be a novel promising agent to target Bcr-Abl and overcome Imatinib resistance in CML patients.

\section{INTRODUCTION}

Chronic myeloid leukemia (CML) is a clonal myeloproliferative disorder that is characterized by high levels of immature white blood cells, which is one of the most understood neoplasms. Annually, newly diagnosed cases of CML in the United States is estimated to be about 4,800 to 5,200 . The estimated prevalence of CML cases in the United States was approximately 25,000 30,000. $\mathrm{CML}$ is defined by the formation and presence of the
Philadelphia $(\mathrm{Ph})$ chromosome, which results from the reciprocal chromosomal translocation $\mathrm{t}(9 ; 22)$ and is represented as $\mathrm{Bcr}-\mathrm{Abl}$ fusion gene. This $\mathrm{Bcr}-\mathrm{Abl}$ fusion protein is found in $\sim 95 \%$ of patients with CML and $20 \sim 30 \%$ of adult patients with acute lymphoblastic leukemia (ALL) [1], a lesser extent Bcr-Abl+ ALL [2]. The $\mathrm{Bcr}-\mathrm{Abl}$ aberrant tyrosine kinase is mainly responsible for malignant transformation by activating multiple signal transduction pathways including Stats, MAPK/ Erk, and PI3K/Akt, which ultimately lead to increased 
survival, proliferation, and escape from apoptosis [3, 4]. For this reason, Bcr-Abl tyrosine kinase has been considered as an important target for CML therapeutics. Meanwhile, Imatinib is considered as the first selective Bcr-Abl tyrosine kinase inhibitor (TKI) for cancer therapy, which interrupts Bcr-Abl oncogenic signaling. Therefore, Imatinib has become the new "gold standard" for the treatment of patients with CML. Especially, 80\% of newly diagnosed patients with chronic-phase CML has shown a complete cytogenetic response to treatment with Imatinib over a median follow-up of 54 months. Although the initial response rates are high, Imatinib fails in up to $40 \%$ of patients because of disease resistance or Bcr-Abl kinase domain mutations, and unacceptable side effects. To circumvent the resistance, more potent TKIs such as Nilotinib and Dasatinib as the second generation TKIs, have been approved. However, these compounds do not show therapeutic activities against all Imatinib-resistant mutants of Bcr-Abl, and finally a long-term tolerablility problem has emerged.

Drug resistance during the Imatinib treatment is mostly related to point mutations occurring within the kinase domain of Bcr-Abl. To date, over 90 different point mutations in the Bcr-Abl kinase domain have been isolated from relapsed CML patients, who are resistant to Imatinib. Among those, the T315I is the most stubborn point mutation having an impact on the binding of Imatinib with Bcr-Abl kinase domain. Also, the T315I is responsible for approximately $15 \%$ of the cases of relapse in CML and Bcr-Abl+ ALL patients on Imatinib therapy [5], and there is high occurrence of E255K and M351T mutations $[6,7]$. Most mutations, except T315I, may be eradicated with the appropriate choice and combinations of second generation tyrosine kinase inhibitors. However, there is still no effective TKI available for CML with the T315I mutation. Considering these facts, the T315I mutation remains a crucial clinical challenge, and it is imperative to develop novel strategies to overcome this resistance.

To resolve these problems, we synthesized and screened a chemical library of novel series of benzothiazole-based inhibitors that are effective against wild-type and T315I mutant Bcr-Abl kinases [8]. Of the benzothiazole-based inhibitors, 1-(6-(2- methoxyphenyl) benzo[d]thiazol-2-yl)-3-(2-(4-methylpiperazin-1-yl)ethyl), HS-543 was identified as the potent Bcr-Abl kinases inhibitor. Here, we investigated whether or not HS-543 possesses anti-cancer activity in BaF3/T315I or BaF3/WT and the molecular mechanisms involved in this process. In this study, our results showed that HS-543 suppressed the Bcr-Abl pathway and induced apoptosis in not only $\mathrm{BaF} 3 / \mathrm{WT}$ cells but also T315I-mutated Bcr-Abl cells with resistance to Imatinib.

\section{RESULTS}

\section{Synthesis of HS-543 and its binding mode to Bcr- Abl}

We identified a novel Bcr-Abl inhibitor, 1-(6-(2-methoxyphenyl)benzo[d]thiazol-2-yl)-3-(2-(4methylpiperazin-1-yl)ethyl) urea or HS-543. As shown in Table 1, HS-543 was further subjected to kinase selectivity profiling against a panel of cancer-related kinases at $1 \mu \mathrm{M}$ concentrations in a high-throughput binding assay (KINOMEscan ${ }^{\circledR}$, Ambit Biosciences, San Diego, CA). Percent of control (POC) determinations were also performed. HS-543 displayed only a high degree of selectivity to Abl but not to other kinases such as Aurora and c-Kit, which are known to have high structural similarities to Abl. The only kinases against which HS543 demonstrated target activity were Abl (POC 1.6) and $\mathrm{Abl} / \mathrm{T} 315 \mathrm{I}$ (POC 3.1). We prepared the receptor model in DFG-in conformation from the X-ray crystal structure of T315I mutant in a complex with PPY-A (PDB code: 2Z60) [9]. Fig. 1 shows the possible binding mode of HS-543 in the ATP binding site of T315I mutant Abl, which was obtained through a docking simulation using the Autodock

A

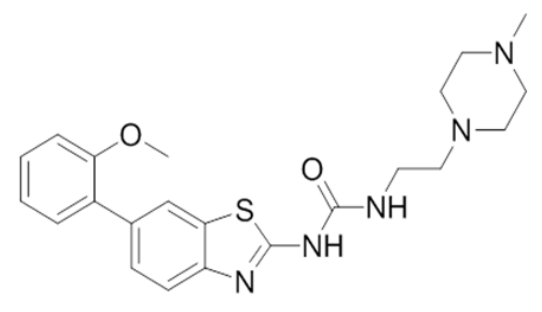

HS-543

1-(6-(2-methoxyphenyl)benzo[d]thiazol-2-yl)-3(2-(4-methylpiperazin-1-yl)ethyl)urea

B

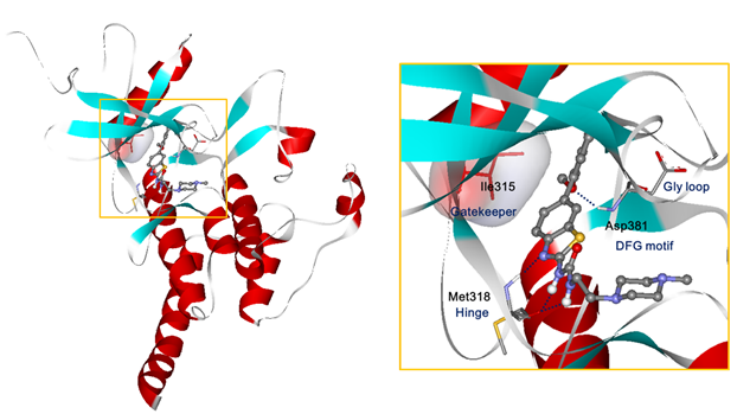

Figure 1: Structure and predicted binding mode of HS-543 with Abl/T315I. (A) Structure of HS-543, 1-(6-(2-methoxyphenyl)benzo[d]thiazol-2-yl)-3-(2-(4-methyl piperazin-1-yl)ethyl)urea. (B) The putative binding mode of HS543 in the Bcr-Abl model. 
Table 1: High-throughput binding assay against a panel of kinases. A panel of 96 kinases was tested at $1 \mu \mathrm{M}$ concentrations in a high-throughput binding assay. Only representative kinases are shown here. Lower percent of control (POC) values represent stronger hits. Values shown are the mean of duplicate measurements.

\begin{tabular}{|c|c|c|c|}
\hline KINOME scan Gene Symbol & Entrez Gene Symbol & POC & Compound Concentration (nM) \\
\hline $\begin{array}{c}\text { ABL1(T315I) } \\
\text {-phosphorylated }\end{array}$ & ABL1 & 3.1 & 1000 \\
\hline ABL1-phosphorylated & ABL1 & 1.6 & 1000 \\
\hline AKT1 & AKT1 & 94 & 1000 \\
\hline AURKA & AURKA & 100 & 1000 \\
\hline BRAF & BRAF & 92 & 1000 \\
\hline CDK11 & CDK19 & 91 & 1000 \\
\hline EGFR & EGFR & 87 & 1000 \\
\hline ERK1 & MAPK3 & 100 & 1000 \\
\hline FAK & PTK2 & 100 & 1000 \\
\hline FGFR1 & FGFR1 & 80 & 1000 \\
\hline GSK3B & GSK3B & 100 & 1000 \\
\hline IKK-beta & IKBKB & 100 & 1000 \\
\hline JAK1(JH1domain-catalytic) & JAK1 & 100 & 1000 \\
\hline KIT & KIT & 58 & 1000 \\
\hline KIT(D816V) & KIT & 86 & 1000 \\
\hline MEK1 & MAP2K1 & 100 & 1000 \\
\hline MET & MET & 74 & 1000 \\
\hline P38-alpha & MAPK14 & 100 & 1000 \\
\hline PAK1 & PAK1 & 88 & 1000 \\
\hline PIK3CA & PIK3CA & 100 & 1000 \\
\hline PIM1 & PIM1 & 98 & 1000 \\
\hline PLK1 & PLK1 & 88 & 1000 \\
\hline ROCK1 & ROCK1 & 100 & 1000 \\
\hline TRKA & NTRK1 & 95 & 1000 \\
\hline
\end{tabular}

program [10]. The docking simulation indicated that C6 methoxyphenyl moiety was surrounded by a hydrophobic pocket, and methyl piperazine moiety in $\mathrm{C} 2$ urea was expected to the solvent front. In the hinge region, the nitrogen of benzothiazole scaffold and the $\mathrm{C} 2$ urea group appeared to form a tight hydrogen bond with the backbone residue of Met318 in the hinge region. Especially, both of the two NHs in the distorted C2 urea group appeared to act as hydrogen bond donors and form hydrogen bonds with aminocarbonyl oxygen of the Met318 backbone. C6 aryl moiety was directed toward the region nearby the gatekeeper and catalytic lysine. The C6 phenyl ring seemed to form favorable van der Waals interactions with the larger mutated gatekeeper, Ile318, without causing steric hindrance. In addition, the oxygen of the 2'-methoxy group in the C6 phenyl ring could contribute to an additional hydrogen bond with the Asp381 backbone.

\section{HS-543 inhibits the proliferation of leukemia cells}

MTS assays were performed to evaluate the effect of HS-543 on the growth of $\mathrm{BaF} 3 / \mathrm{WT}$ and $\mathrm{BaF} 3 / \mathrm{T} 315 \mathrm{I}$ cells. The cells were treated with various concentrations of HS543 and Imatinib for $48 \mathrm{~h}$. As shown in Fig. 2A, both HS543 and Imatinib treatments reduced the cell viability of the $\mathrm{BaF} 3 / \mathrm{WT}$ cells; $\mathrm{IC}_{50}$ values were $0.33 \mu \mathrm{M}$ for Imatinib and $0.116 \mu \mathrm{M}$ for HS-543. As shown in Fig. 2B, HS-543 significantly reduced the cell viability of $\mathrm{BaF} 3 / \mathrm{T} 315 \mathrm{I}$ cells $\left(\mathrm{IC}_{50}=0.33 \mu \mathrm{M}\right)$, while Imatinib showed little effect $\left(\mathrm{IC}_{50}=10 \mu \mathrm{M}\right)$. These data suggest that HS-543 exhibits the potent inhibitory activity in T315I mutated Bcr-Abl 
cells with the resistance to Imatinib, as well as Bcr-Abl expressing leukemic cells.

\section{HS-543 inhibits Bcr-Abl signaling pathway in BaF3/T315I cells}

To test if the antiproliferative effects of HS-543 were dependent on the inhibition of Bcr-Abl activity, phosphorylation of Bcr-Abl and its respective downstream signals, Crkl and Stat5, were measured by western blotting. As shown in Fig. 3A and 3B, HS-543 strongly inhibited the phosphorylation of Bcr-Abl $\left(\mathrm{Tyr}^{177}\right)$ in $\mathrm{BaF} 3 /$ T315I cells. Likewise, the phosphorylation levels of Crkl $\left(\mathrm{Tyr}^{207}\right)$ and Stat5 $\left(\mathrm{Tyr}^{694}\right)$ were effectively suppressed in a dose- and time-dependent manner. In contrast, Imatinib did not alter the phosphorylation levels of Bcr-Abl, Crkl and Stat5 in BaF3/T315I cells. These results were confirmed by a confocal fluorescent microscopy (Fig. 3C).

\section{HS-543 induces apoptotic cell death in BaF3/ T315I cells}

In order to determine whether the anti-cancer effect of HS-543 in BaF3/T315I cells was associated with

A

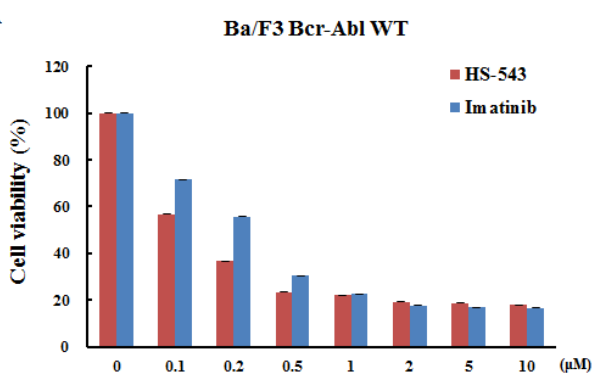

B

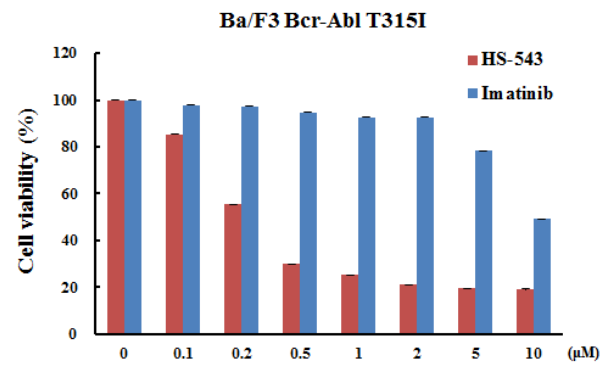

Figure 2: Cytotoxic effect of HS-543 in leukemia cells. Cytotoxic effects of leukemia cells treated with HS-543 and Imatinib were measured using the MTS assay. Cells were seeded in 96-well culture plates, and were treated with various concentrations of HS-543 and Imatinib. Cells were subjected to an MTS assay following incubation for $48 \mathrm{~h}$. Data are represented as mean \pm S.D. from triplicate wells. the induction of apoptosis, we performed several cellbased apoptosis assays. We first assessed the cell cycle distribution by a flow cytometric analysis. The cells were collected, stained with PI, and analyzed by flow cytometry after they were incubated with HS-543 for various doses and times. As shown in the Fig. 4A, HS-543 increased the number of cells in the sub $G_{1}$ phase, associated with early apoptosis without changes of the cell cycle arrest. To characterize nuclear morphology, the nuclei were stained with Hoechst, and a TUNEL assay was performed. As a result, the cells treated with HS-543 were presented with more prominent morphological features of apoptotic cells, such as bright nuclear condensation by Hoechst staining (Fig. 4B). HS-543-induced apoptosis was confirmed by detecting DNA fragmentation using TUNEL staining. Cells were treated with HS-543 for $24 \mathrm{~h}$ (Fig. 4C, **p $<0.001)$. Furthermore, HS-543 significantly increased the expression of cleaved PARP and cleaved caspase-3, apoptosis-related molecules in BaF3/T315I cells (Fig. 4D), compared with Imatinib. An increase of cleaved caspase-3 was also confirmed by immunofluorescence after treating with $1 \mu \mathrm{M}$ HS-543 in BaF3/T315I cells for $24 \mathrm{~h}$ (Fig. 4E).

A B

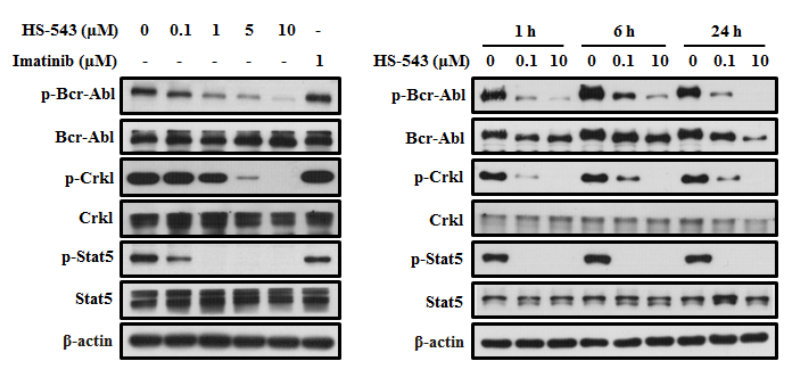

C

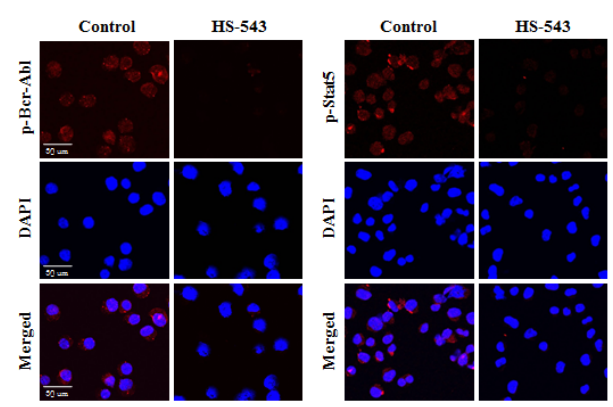

Figure 3: Effect of HS-543 on the Bcr-Abl signaling pathway in BaF3/T315I cells. (A) Cell were treated with HS-543 $(0.01-10 \mu \mathrm{M})$ or Imatinib $(1 \mu \mathrm{M})$ for $24 \mathrm{~h}$. Western blot experiments for Bcr-Abl, Crkl, and Stat5, and the respective phosphorylated proteins were performed with cell lysates. (B) Cells were treated with HS-543 for time-dependent manner. (C) After BaF3/T315I cells were treated with $10 \mu \mathrm{M}$ HS-543 for $3 \mathrm{~h}$, p-Bcr-Abl and p-Stat5 levels were detected by confocal fluorescent microscopy (200× magnification). 


\section{HS-543 induces mitochondria-dependent apoptosis in $\mathrm{BaF} 3 / \mathrm{T} 315 \mathrm{I}$ cells}

Loss of mitochondrial membrane potential (MMP) induces mitochondrial permeability transition and cytosolic translocation of apoptotic proteins [11]. Thus, we measured MMP and apoptosis in HS-543-treated BaF3/T315I cells using TMRE. As shown in Fig. 5A, HS-543 significantly reduced the fluorescence intensity reflecting MMP $(* p<0.01)$. Since MMP can trigger the release of mitochondrial cytochrome $c$ into the cytosol and induces mitochondria-mediated protein families such as Mcl-1, survivin and Bax, we investigated their expression by HS-543 in BaF3/T315I cells [9]. As shown in Fig. $5 \mathrm{~B}$, we observed that the treatment of HS-543 increased cytochrome $c$ release by immunostaining and western blotting. In addition, HS-543 increased the expression of Bax and decreased the expression of the anti-apoptotic proteins survivin and Mcl-1 (Fig. 5C). These results showed that HS-543 induced apoptosis through change of mitochondria-related proteins in BaF3/T315I cells.
A

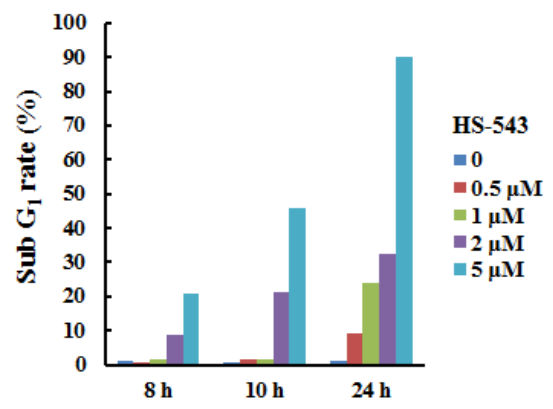

B

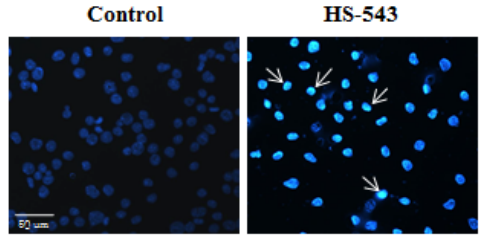

C

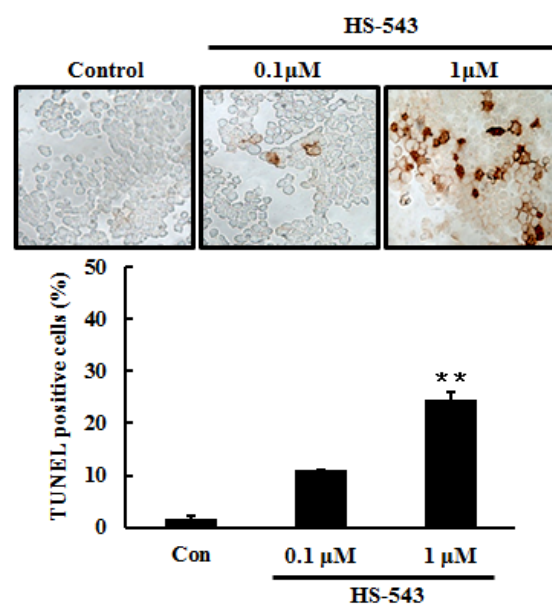

D

$\mathbf{E}$
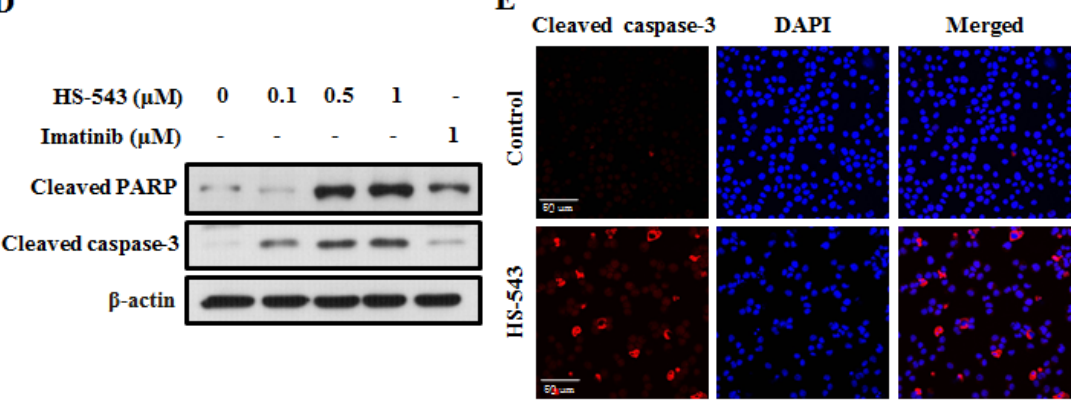

Figure 4: Effect of HS-543 on apoptosis of BaF3/T315I cells. (A) Cells were incubated with HS-543 for various dose and times. Control and treated cells were collected, stained with PI, and analyzed by flow cytometry. (B) Cell were treated with HS-543 (1 $\mu \mathrm{M}$ ) for $24 \mathrm{~h}$. Then, cells were fixed in ice-cold 4\% PFA, washed with PBS, and then stained with $1 \mu \mathrm{g} / \mathrm{ml}$ Hoechst 33342 for 20 min at room temperature. The stained cells were examined under immunofluorescence for evidence of nuclear fragmentation (400× magnification). $(\mathrm{C})$ Cells were treated with HS-543 for $24 \mathrm{~h}$ and were performed with TUNEL assay. Data are represented as mean \pm S.D. from triplicate wells. ${ }^{* *} p<0.001$ as compared to control. (D) For detection of expression of cleaved PARP and cleaved caspase-3, the cells were treated with HS-543 and Imatinib for $36 \mathrm{~h}$. (E) (E) Immunofluorescence of cleaved caspase-3 after treatment of HS-543 (1 $\mu \mathrm{M})$ in BaF3/T315I cells for $24 \mathrm{~h}(400 \times$ magnification). 


\section{HS-543 inhibits tumor growth in mouse xenograft models}

We extended our study to an in vivo mouse xenograft model. After inoculation with BaF3/T315I cells, mice were intraperitoneally injected with HS-543 at doses of 30 and $50 \mathrm{mg} / \mathrm{kg}$, and Imatinib at a dose of $50 \mathrm{mg} /$ $\mathrm{kg} 5$ times a week for 14 days. While Imatinib treatment didn't show significant anticancer effect in this BaF3/ T315I cell xenograft model, HS-543 potently inhibited the progression of tumor growth and more visible and significant on day 14 as compared with the control group $(* p<0.01$, Fig. 6A). Isolated tumor weight was also remarkably lower in the HS-543 treated group than in the control group (Fig. 6B, $* p<0.01$ ). No significant changes in body weight or adverse effect were observed in all groups. To further confirm whether HS-543 inhibits tumor growth through the induction of apoptosis and inhibition of proliferation, we identified the expression of cleaved caspase-3 and PCNA in the isolated tumor tissues. As expected, the treatment with HS-543 increased the expression of cleaved caspase-3 and decreased for PCNA in the HS-543 treated group as compared to the control and Imatinib groups (Fig. 7A). Furthermore, the treatment with HS-543 decreased the phosphorylation of

$\mathbf{A}$
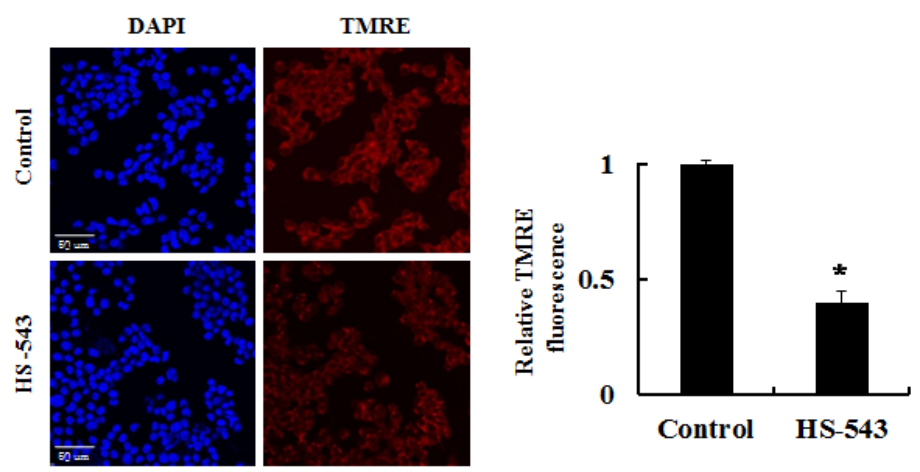

B
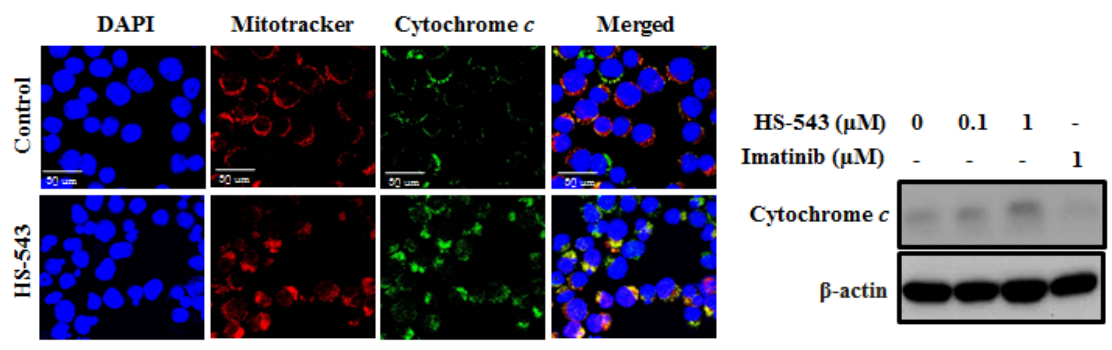

C

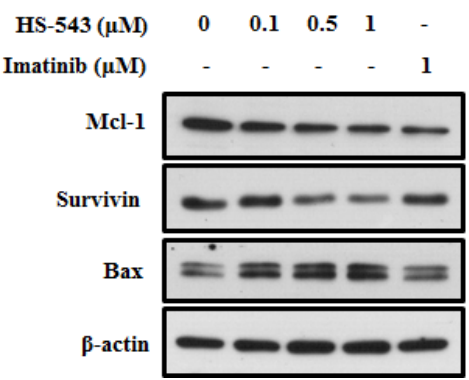

Figure 5: Effect of HS-543 on mitochondria-related apoptosis of BaF3/T315I cells. (A) BaF3/T315I cells were treated with HS-543 at $1 \mu \mathrm{M}$ for $12 \mathrm{~h}$ and were stained with TMRE for $30 \mathrm{~min}$ at $37^{\circ} \mathrm{C}$ and analyzed with a confocal fluorescent microscopy (400× magnification). ${ }^{*} p<0.01$ as compared to control. (B) BaF3/T315I cells were treated with HS-543 at $1 \mu \mathrm{M}$ for $10 \mathrm{~h}$ and were stained with mitotracker and cytochrome $c$. Localization of cytochrome $c$ in the cytosol by HS- 543 was analyzed with a confocal fluorescent microscopy (1200× magnification). For western blotting, BaF3/T315I cells were treated with 0.1 and $1 \mu \mathrm{M}$ HS-543 together with $1 \mu \mathrm{M}$ Imatinib for 12 h. (C) The expression of Mcl-1, survivin and Bax were assayed by western blot in BaF3/T315I cells. Cells were treated with HS-543 and Imatinib for $12 \mathrm{~h}$. 
p-Bcr-Abl and p-Stat5; thus, regulating many different events involved in cell survival and proliferation (Fig. 7B). Taken together, these results demonstrate that HS-543 has a potent antitumor efficacy in mouse xenograft model bearing BaF3/T315I cells.

\section{DISCUSSION}

In $95 \%$ of CML cases, the product of a reciprocal translocation between chromosomes 9 and 22, the Philadelphia chromosome, is detected, which is characterized by the presence of $\mathrm{Bcr}-\mathrm{Abl}$ fusion gene, representing a subtype of leukemia with poor prognosis, rapidly acquiring resistance to the Imatinib treatment during therapy $[12,13]$. Imatinib is the molecular targeted agent that selectively inhibits the Bcr-Abl tyrosine kinase activity. To date, Imatinib has been recognized as a

$\mathbf{A}$

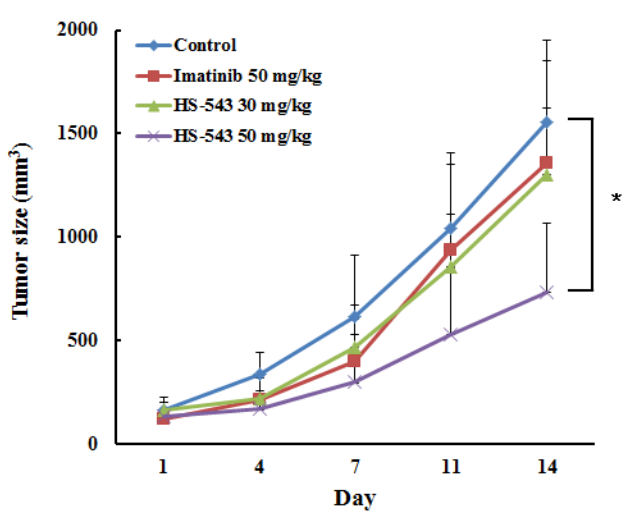

B

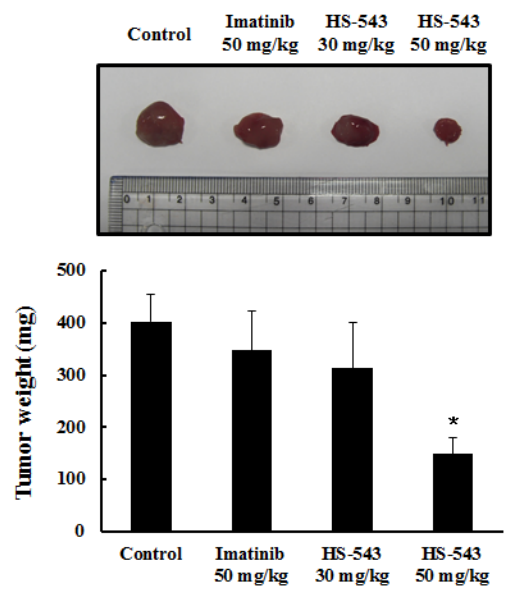

Figure 6: In vivo anticancer effect of HS-543 in mouse xenograft model. (A) Tumor growth of BaF3/T315I xenograft in $\mathrm{BALB} / \mathrm{c}$ nude mice. All mice were subjected to implantation in the flank by a subcutaneous injection of BaF3/T315I $\left(1 \times 10^{5}\right.$ cells $/ 200 \mu \mathrm{L}$ PBS). HS-543 (30 and $50 \mathrm{mg} / \mathrm{kg}$ ) and Imatinib 50 $\mathrm{mg} / \mathrm{kg}$ was intraperitoneally injected 5 times a week for 14 days. Tumor size was measured every 2 days. Data were represented as mean \pm S. D. $(n=5)$. (B) After mouse sacrifice, representative photographs and mean weights of the isolated tumors. $* p<0.01$ as compared to control. revolutionary treatment of CML, due particularly to the durable remission most chronic-phase patients experience. Also, Imatinib inhibits Bcr-Abl kinase with $50 \%$ inhibitory concentration values of $0.1-0.5 \mu \mathrm{M}$ in cell-based assays $[14,15]$. Targeted therapy to inhibit the oncogene Bcr-Abl seems to be somewhat successful. However, Imatinib resistance often occurs in patients especially in CML accelerated phase and blast crisis, and almost unrelievedly occurs in patients with the BcrAbl expression, showing shortcomings of the therapy. According to Imatinib resistance, amplification and mutation of Bcr-Abl is believed to be the predominant. To overcome this acquired resistance to Imatinib, a lot of researchers have developed a new, second-generation ATP-competitive Abl kinase inhibitors, such as AMN107, Dasatinib, INNO-406 and PD166326, which have stronger affinities for the ATP-binding site of Bcr-Abl kinase than Imatinib, and has shown partial effect for Imatinibresistant patients [16-20]. Although these novel inhibitors can effectively inhibit the phosphorylation of the mutated Bcr-Abl (E255K and M351T), they showed little effect on Bcr-Abl/T315I [21]. Therefore, innovative treatment is necessary to over-ride TKI-resistant mutations, or to promote elimination of Bcr-Abl harboring cells in patients. The principal objective of this study is to identify

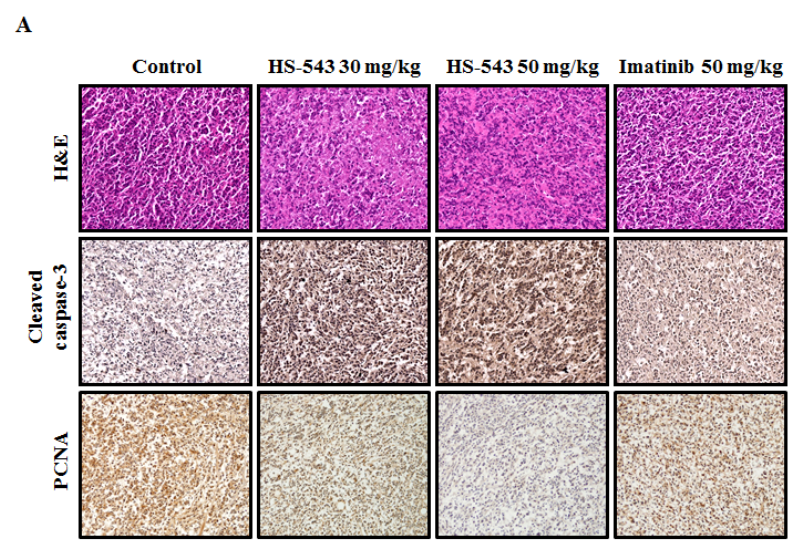

B

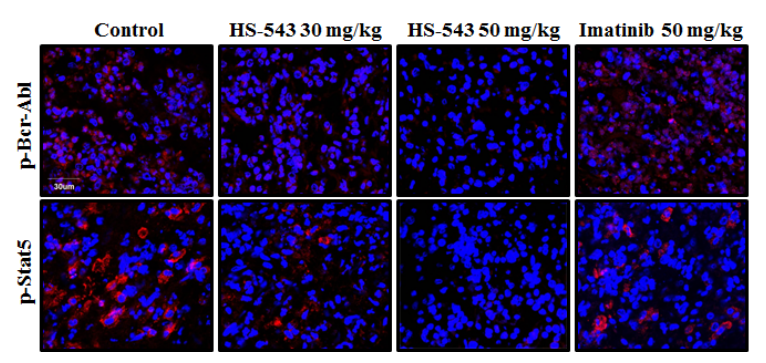

Figure 7: Effect of HS-543 on proliferation and apoptosis in isolated tumor from mouse xenograft model. (A) Tumors were excised and processed for immnunohistochemistry to detect cleaved caspase-3 and PCNA including H\&E staining. (B) Tumors were stained with p-Bcr$\mathrm{Abl}$ and p-Stat5 and were detected by confocal fluorescent microscopy (400× magnification). 
the effective targeted chemotherapy against CML cells carrying T315I-mutant Bcr-Abl that confers resistance to Imatinib. In this study, we demonstrated that HS-543, a novel Bcr-Abl TKI, had high affinity for the ATP-binding site, resulting in high specificity to BaF3/T315I (Table 1). In light of these results, we investigated whether HS-543 had potent activity on Bcr-Abl target inhibition in $\mathrm{BaF} 3$ / WT and BaF3/T315I CML cell lines. HS-543 potently inhibited the proliferation in both $\mathrm{BaF} 3 / \mathrm{WT}\left(\mathrm{IC}_{50}=0.116\right.$ $\mu \mathrm{M})$ and $\mathrm{BaF} 3 / \mathrm{T} 315 \mathrm{I}\left(\mathrm{IC}_{50}=0.33 \mu \mathrm{M}\right)$ cells. However, Imatinib did not inhibit the proliferation in BaF3/T315I cells, since $\mathrm{IC}_{50}$ values were more than $10 \mu \mathrm{M}$.

Bcr-Abl activates multiple downstream signaling pathways, including Stat5 pathway, which contribute to leukemic cell proliferation and survival [22, 23]. Also, the Stat5 activation is mediated by the adaptor protein Crkl $[24,25]$. In addition, Stat5 activity appears to play a major role in the antiapoptotic and proliferative abilities of Bcr-Abl transformed cells [22, 23]. Previous studies have established phosphorylation of Stat5 as an indicator of Bcr-Abl activity [26]. Therefore, we identified whether HS-543 could inhibit Bcr-Abl signaling pathway. As expected, HS-543 distinctly inhibited the phosphorylation of Bcr-Abl, which indicates decreased Abl kinase activity, as well as a pronounced inhibition of Bcr-Abl downstream target Stat5 phosphorylation in $\mathrm{BaF} 3 / \mathrm{T} 315 \mathrm{I}$ cells. Also, phosphorylation of another well-known Bcr-Abl downstream target Crkl was obviously reduced after the treatment with HS-543. Interestingly, Imatinib inhibited the phosphorylation of Bcr-Abl pathways, such as BcrAbl, Stat5, and Crkl in BaF3/WT cells, but not in BaF3/ T315I cells. These results revealed that the decrease of phosphorylation of Crkl and Stat5 by HS-543 in the cells with the highly resistant mutation T315I was significant, which indicate an effective inhibition of Bcr-Abl carrying this highly resistant mutation.

Also, HS-543 induces mitochondrial apoptosis in $\mathrm{BaF} 3 / \mathrm{T} 3151$ cells. In fact, apoptosis has been noticed to be associated with a loss of mitochondrial membrane potential, which may correspond to the opening of an outer membrane pore, leading to the release of cytochrome $c$ into the cytoplasm [27]. Also, the release of cytochrome $c$ induces the expression of various pro-apoptotic proteins in cytoplasm [28]. Especially, Bax initiates a mitochondrial permeability shift and induces apoptosis by moving from the cytosol to the mitochondrial membrane [29]. And, Mcl-1 and survivin as anti-apoptotic proteins have been reported to bind to caspases to inhibit apoptosis signaling [30]. More importantly, Bcr-Abl inhibits apoptosis through regulating Bcl-2 family members [31], increasing the expression of antiapoptotic Bcl-2 family members, such as Bax and Bcl-2 through activation of the transcription factor Stat5 [32-34]. Additionally, Bcr-Abl has been shown to prevent mitochondrial cytochrome $c$ release through a posttranslational mechanism [35]. Accordingly, we assumed that HS-543 would affect the mitochodrial membrane potential and induce apoptosis since HS-543 showed a high specific inhibition against Bcr-Abl. Indeed, we observed that HS-543 increased cytochrome $c$ release and decreased the expression of Mcl-1 and survivin, whereas increased the expression of Bax. These changes led to an increase the expression of cleaved caspase- 3 and cleavage of PARP. These events were supported by in vivo results, showing that HS-543 inhibited the tumor growth and induced apoptosis by increasing the expression of cleaved caspase- 3 in tumor tissues of BaF3/T315I cells xenograft mice. However, Imatinib did not change the expression of apoptosis-related molecules in vitro and in vivo. Overall, HS-543 showed to induce apoptosis via an intrinsic mitochondria-dependent pathway in BaF3/T315I cells, suggesting that the apoptosis effect by HS-543 may be achieved by inhibition of Bcr-Abl signaling pathways.

In conclusion, our results showed that the novel selective Bcr-Abl TKI, HS-543, had potent anticancer activity against the cells bearing wild type and T315I mutant type Bcr-Abl. Given the mechanism of action and the promising activity of HS-543 against Imatinib-resistant CML cells, our results provide an important vision to be considered for future clinical investigations in $\mathrm{CML}$ patients with Imatinib resistance. Here, we suggest that HS-543 may have great potential in overcoming T315I mutation-induced Imatinib resistance.

\section{METERIALS AND METHODS}

\section{Cells and materials}

The BaF3/WT and BaF3/T315I cells were kindly provided by Dr. Deininger (Huntsman Cancer Institute, Salt Lake City, UT), which inducibly expressed wildtype Bcr-Abl and Bcr-Abl with the T315I mutation, respectively. BaF3/WT and $\mathrm{BaF} 3 / \mathrm{T} 315 \mathrm{I}$ cells were grown in Roswell Park Memorial Institute Media 1640 (RPMI 1640), containing 10\% fetal bovine serum (FBS) and $1 \%$ penicillin/streptomycin. RPMI 1640; FBS and penicillin/streptomycin were purchased from GIBCO (Grand Island, NY). Imatinib was purchased from LC laboratories (Woburn, MA), and 3-(4,5-dimethylthiazol2-yl)-5-(3-carboxymethoxyphenyl)-2-(4-sulfophenyl)-

2H-tetrazolium, inner salt (MTS) were purchased from Promega (Madison, WI).

\section{Enzyme binding assay}

The enzyme binding assay of HS-543 was performed against a panel of 96 kinases using KINOMEscan assays at the Ambit Biosciences Corp (San Diego, CA). In brief, HS-543 was screened at $1 \mu \mathrm{M}$ and results for primary screen binding interactions were reported as ' $\% \mathrm{Ctrl}$ '. \% Ctrl Calculation $=($ HS-543 signal - positive control signal 
/ negative control signal - positive control signal) x100, where negative control is DMSO $(100 \% \mathrm{Ctrl})$, and positive control is control compound $(0 \% \mathrm{Ctrl})$. Accordingly, lower percent of control (POC) values represent stronger hits [8].

\section{Cell viability assay}

Cell viability of corresponding compounds was determined by MTS assay. The cells were seeded and treated onto 96-well plates at a density of $1 \times 10^{3}$ cells per well and incubated at $37^{\circ} \mathrm{C}$ for $48 \mathrm{~h}$. The cells were treated with either HS-543 or Imatinib at the indicated concentrations $(0.001-10 \mu \mathrm{M})$. Then, $20 \mu \mathrm{L}$ of MTS labeling mixture $(1 \mathrm{~mL}$ of $\mathrm{MTS} / 50 \mu \mathrm{L}$ of phenazine methosulfate [PMS]) was added to each well. After incubation for $4 \mathrm{~h}$, optical density (OD) was determined using a microplate reader by measuring the absorbance at wavelengths of $540 \mathrm{~nm}$.

\section{Western blotting}

After the cells were treated with various concentrations of either HS-543 or Imatinib and incubated at $37^{\circ} \mathrm{C}$ for various times, they were collected and washed with cold phosphate-buffered saline (PBS). Then, the cells were lysed with a RIPA buffer (BIOSESANG, Korea) containing protease and phosphatase inhibitor cocktails (GenDEPOT, Barker, TX). The proteins were resolved by sodium dodecyl sulfate-polyacrylamide gel electrophoresis (SDS-PAGE), and transferred onto the nitrocellulose membranes. The blots were immunostained with the appropriate primary antibodies followed by secondary antibodies conjugated to horseradish peroxidase. Antibody binding was detected with an enhanced chemiluminescence reagent (Bio-Rad. Hercules, CA). Primary monoclonal antibodies against the following factors were used; p-Bcr-Abl (Tyr $\left.{ }^{177}\right)$, p-Crkl $\left(\mathrm{Tyr}^{207}\right)$, p-Stat5 (Tyr $\left.{ }^{694}\right)$, Bcr-Abl, Crkl, Stat5, cleaved PARP, cleaved caspase-3, cytochrome $c$, survivin, Mcl-1, Bax and $\beta$-actin (Cell Signaling Technology, Danvers, MA). Secondary antibodies were purchased from Santa Cruz Biotechnology (Dallas, TX).

\section{Immunofluorescence}

BaF3/T315I cells were plated in 48-well plates with RPMI medium and treated with $1 \mu \mathrm{M}$ HS-543 for various times. The cells were then suspended on poly-1lysine-coated slides, followed by Shandon Cytospin 3 (Akribis Scientific, Cheshire, WA) for $3 \mathrm{~min}$ at $1000 \mathrm{rpm}$. Thereafter, the cells were fixed in 4\% paraformaldehyde (PFA) for $15 \mathrm{~min}$ at room temperature and washed twice with PBS. The cells were blocked in 5\% horse and goat serum in PBS for $1 \mathrm{~h}$ at room temperature, and then incubated in a humidified chamber at $4{ }^{\circ} \mathrm{C}$ overnight with primary antibodies including rabbit anti-p-Bcr-Abl $\left(\mathrm{Tyr}^{177}\right)$ and p-Stat5 (Tyr $\left.{ }^{694}\right)$ (Cell Signaling Technology). After washing twice with PBS, the cells were incubated with rabbit tetramethyl rhodamine isothiocyanate (TRITC) secondary antibody (Dianova, Germany) for $1 \mathrm{~h}$ at room temperature. They were also stained with 4,6-diamidino2-phenylindole (DAPI) to visualize the nuclei. The slides were then washed twice with PBS and covered with DAKO (Carpinteria, CA) before viewing with a confocal laser scanning microscope (Olympus, Japan).

Also, after deparaffinization, immunostaining was performed, using 8 - $\mu \mathrm{m}$-thick sections of the tumor samples. The tissue sections were blocked with a normal goat or horse serum (Vector Laboratories. Burlingame, CA) for $1 \mathrm{~h}$, and incubated at $4^{\circ} \mathrm{C}$ overnight in 1:50 dilutions of p-Bcr-Abl (Tyr $\left.{ }^{177}\right)$ and p-Stat5 $\left(\mathrm{Tyr}^{694}\right)$ (Cell Signaling Technology). After washing twice with PBS, the cells were incubated with rabbit TRITC secondary antibody for $1 \mathrm{~h}$ at room temperature. They were also stained with DAPI to visualize the nuclei. The slides were then washed twice with PBS and covered with DAKO before viewing with a confocal laser scanning microscope.

\section{Cell cycle arrest}

$\mathrm{BaF} 3 / \mathrm{T} 315 \mathrm{I}$ cells were plated in $10 \mathrm{~cm}$ dishes with RPMI medium and treated with HS-543 at the indicated concentrations $(0.5-5 \mu \mathrm{M})$. The cells were collected and fixed in cold $70 \%$ ethanol at $-25^{\circ} \mathrm{C}$ overnight. After washing with PBS, the cells were subsequently stained with $50 \mu \mathrm{g} / \mathrm{mL}$ propidium iodide (PI) and $100 \mu \mathrm{g} / \mathrm{mL}$ RNase A for 30 min at room temperature in the dark; then a flow cytometric analysis was performed to determine the percentage of cells in specific phases of sub $G_{1}$ using a FACS Calibur flow cytometer (BD Biosciences. San Jose, CA). Flow cytometric data was analyzed using FlowJo software (Tree Star. Ashland, OR).

\section{Hoechst staining and TUNEL staining}

BaF3/T315I cells were plated in 48-well plates with RPMI medium and treated with HS-543 (1 $\mu \mathrm{M})$. The cells were then suspended on poly-1-lysine-coated slides, followed by Shandon Cytospin 3 for 5 min at 1000 $\mathrm{rpm}$. They were then fixed in 4\% PFA for $15 \mathrm{~min}$ at room temperature, washed with PBS, and stained with $1 \mu \mathrm{g} / \mathrm{mL}$ Hoechst 33342 (Cell Signaling Technology) for $20 \mathrm{~min}$ at room temperature. The stained cells were examined under a fluorescence microscope for evidence of nuclear fragmentation. Terminal deoxynucleotidyl transferasemediated nick end labeling (TUNEL) was subsequently performed using a TUNEL kit (Merck Millipore, Temecula, CA) in accordance to the manufacturer's instructions. 


\section{Measurement of mitochondrial membrane potential}

Mitochondrial membrane potential (MMP, $\Delta \psi m$ ) was assessed using Multi-Parameter Apoptosis Assay Kit (Cayman, Ann Arbor, MI). BaF3/T315I cells were treated with $1 \mu \mathrm{M}$ HS-543 for $12 \mathrm{~h}$. The cells were incubated with tetramethylrhodamine ethyl ester (TMRE), and DAPI staining at dark room condition. The cells were then suspended on poly-l-lysine-coated slides, followed by Shandon Cytospin 3 for $3 \mathrm{~min}$ at $1000 \mathrm{rpm}$. The slides were covered with DAKO before viewing with a confocal laser scanning microscope.

\section{Analysis of cytochrome $c$ localization}

$\mathrm{BaF} 3 / \mathrm{T} 315 \mathrm{I}$ cells were treated with $1 \mu \mathrm{M}$ HS-543 for $10 \mathrm{~h}$. To label the mitochondria, the cells were incubated with $100 \mathrm{nM}$ mitochondrion-specific dye (MitoTracker ${ }^{\circledR}$ Red FM; Molecular Probes Inc., Eugene, OR) for 45 min at $37^{\circ} \mathrm{C}$ prior to fixation. The cells were then suspended on poly-l-lysine-coated slides, followed by Shandon Cytospin 3 for $3 \mathrm{~min}$ at $1000 \mathrm{rpm}$. They were then fixed in 4\% PFA for $15 \mathrm{~min}$ at room temperature and washed with PBS. The cells were incubated at $4^{\circ} \mathrm{C}$ overnight with cytochrome $c$ antibody (Santa Cruz Biotechnology). After washing twice with PBS, the cells were incubated with mouse fluorescein isothiocyanate (FITC) secondary antibody (Dianova). The cells were also stained with DAPI to visualize the nuclei. The slides were then washed twice with PBS and covered with DAKO before viewing with a confocal laser scanning microscope.

\section{Tumor xenograft study}

All animal experiments were performed in accordance with the guidelines of the INHA Institutional Animal Care and Use Committee (INHA IACUC at Inha University Medical School, under the authority of project number INHA 130318-197. The cells were harvested and mixed in PBS ( $200 \mu \mathrm{L} /$ mouse). Six weeks old male BALB/c nude mice (Orient Bio, Korea) were inoculated with $1 \times 10^{5}$ cells in the flank. When the tumor size reached approximately $50-100 \mathrm{~mm}^{3}$, they were randomly divided into 4 groups of 5 mice. Then, these mice were given HS-543 or Imatinib by intraperitoneally 5 times a week for 14 days. The treat group was fed with either HS-543 (30 mg/kg and $50 \mathrm{mg} / \mathrm{kg}$ ) or Imatinib $(50 \mathrm{mg} / \mathrm{kg})$, and the control group was fed vehicle. Tumor size and body weight were measured every 2 days. Tumor size was calculated using the formula $0.5 \times$ length $\times$ width $^{2}$

\section{Immunohistochemisry}

The tissue sections were blocked with normal goat or horse serum (Vector Laboratories) for $1 \mathrm{~h}$, and incubated at $4^{\circ} \mathrm{C}$ overnight in 1:50 dilutions of cleaved caspase-3 and proliferating cell nuclear antigen (PCNA) (Cell Signaling Technology). The sections were then incubated with biotinylated secondary antibodies (1:100) for $1 \mathrm{~h}$. The sections were visualized by an avidin-biotin peroxidase complex solution using an ABC kit (Vector Laboratories), which were then washed in PBS and developed with a diaminobenzidine tetrahydrochloride substrate for 15 min and then counterstained with hematoxylin. At least 3 random fields of each section were examined at $400 \times$ magnification and analyzed using a computer image analysis system (Media Cybernetics. Rockville, MD).

\section{Statistical analysis}

Data were expressed as the mean \pm standard deviation (S.D.). Statistical analysis was performed using ANOVA and unpaired Student's $t$-tests. The differences were considered statistically significant when $p<0.01$.

\section{ACKNOWLEDGEMENTS}

This research was supported by the National Research Foundation of Korea grant (2012R1A2A2A010), Medical Research Center (2014009392) funded by MSIP, the Institute for Basic Science (IBS-R010-G1), and a grant (A110076) from the Korea Health Technology R\&D Project, Ministry of Health and Welfare, Republic of Korea.

\section{REFERENCES}

1. Melo JV and Barnes DJ. Chronic myeloid leukaemia as a model of disease evolution in human cancer. Nat Rev Cancer. 2007; 7(6):441-453.

2. Nguyen T, Dai Y, Attkisson E, Kramer L, Jordan N, Nguyen N, Kolluri N, Muschen M and Grant S. HDAC inhibitors potentiate the activity of the $\mathrm{BCR} / \mathrm{ABL}$ kinase inhibitor KW-2449 in imatinib-sensitive or -resistant BCR/ $\mathrm{ABL}+$ leukemia cells in vitro and in vivo. Clin Cancer Res. 2011; 17(10):3219-3232.

3. Lu Z, Jin Y, Qiu L, Lai Y and Pan J. Celastrol, a novel HSP90 inhibitor, depletes Bcr-Abl and induces apoptosis in imatinib-resistant chronic myelogenous leukemia cells harboring T315I mutation. Cancer Lett. 2010; 290(2):182191.

4. Demidenko ZN, An WG, Lee JT, Romanova LY, McCubrey JA and Blagosklonny MV. Kinase-addiction and bi-phasic sensitivity-resistance of Bcr-Abl- and Raf-1expressing cells to imatinib and geldanamycin. Cancer Biol 
Ther. 2005; 4(4):484-490.

5. Martinelli G, Iacobucci I, Soverini S, Palandri F, Castagnetti F, Rosti $G$ and Baccarani M. Nilotinib: a novel encouraging therapeutic option for chronic myeloid leukemia patients with imatinib resistance or intolerance. Biologics. 2007; 1(2):121-127.

6. Gorre ME, Mohammed M, Ellwood K, Hsu N, Paquette R, Rao PN and Sawyers CL. Clinical resistance to STI571 cancer therapy caused by BCR-ABL gene mutation or amplification. Science. 2001; 293(5531):876-880.

7. Blagosklonny MV. STI-571 must select for drug-resistant cells but 'no cell breathes fire out of its nostrils like a dragon'. Leukemia. 2002; 16(4):570-572.

8. Hong S, Kim J, Yun SM, Lee H, Park Y, Hong SS, Hong S. Discovery of new benzothiazole-based inhibitors of breakpoint cluster region-Abelson kinase including the T315I mutant. J Med Chem. 2013; 56(9):3531-3545.

9. Zhou T, Parillon L, Li F, Wang Y, Keats J, Lamore S, Xu Q, Shakespeare W, Dalgarno D and Zhu X. Crystal structure of the T315I mutant of AbI kinase. Chem Biol Drug Des. 2007; 70(3):171-181.

10. Morris GM, Goodsell DS, Halliday RS, Huey R, Hart WE, Bellew RK and Olson AJ. Automated Docking Using a Lamarckian Genetic Algorithm and an Empirical Binding Free Energy Function. J Comput Chem. 1998; 19(14):16391662.

11. Kaufmann SH and Hengartner MO. Programmed cell death: alive and well in the new millennium. Trends Cell Biol. 2001; 11(12):526-534.

12. Lim YP, Diong LS, Qi R, Druker BJ and Epstein RJ. Phosphoproteomic fingerprinting of epidermal growth factor signaling and anticancer drug action in human tumor cells. Mol Cancer Ther. 2003; 2(12):1369-1377.

13. Hu Y, Swerdlow S, Duffy TM, Weinmann R, Lee FY and Li S. Targeting multiple kinase pathways in leukemic progenitors and stem cells is essential for improved treatment of $\mathrm{Ph}+$ leukemia in mice. Proc Natl Acad Sci U S A. $2006 ; 103(45): 16870-16875$.

14. Druker BJ, Tamura S, Buchdunger E, Ohno S, Segal GM, Fanning S, Zimmermann J and Lydon NB. Effects of a selective inhibitor of the Abl tyrosine kinase on the growth of Bcr-Abl positive cells. Nat Med. 1996; 2(5):561-566.

15. Beran M, Cao X, Estrov Z, Jeha S, Jin G, O'Brien S, Talpaz M, Arlinghaus RB, Lydon NB and Kantarjian H. Selective inhibition of cell proliferation and BCR-ABL phosphorylation in acute lymphoblastic leukemia cells expressing Mr 190,000 BCR-ABL protein by a tyrosine kinase inhibitor (CGP-57148). Clin Cancer Res. 1998; 4(7):1661-1672.

16. Talpaz M, Shah NP, Kantarjian H, Donato N, Nicoll J, Paquette R, Cortes J, O'Brien S, Nicaise C, Bleickardt E, Blackwood-Chirchir MA, Iyer V, Chen TT, Huang F, Decillis AP, Sawyers CL, et al. Dasatinib in imatinibresistant Philadelphia chromosome-positive leukemias. N
Engl J Med. 2006; 354(24):2531-2541.

17. Kaur P, Feldhahn N, Zhang B, Trageser D, Müschen M, Pertz V, Groffen J and Heisterkamp N. Nilotinib treatment in mouse models of P190 Bcr/Abl lymphoblastic leukemia. Mol Cancer. 2007; 6:67.

18. Morinaga K, Yamauchi T, Kimura S, Maekawa $\mathrm{T}$ and Ueda T. Overcoming imatinib resistance using Src inhibitor CGP76030, Abl inhibitor nilotinib and Abl/Lyn inhibitor INNO-406 in newly established K562 variants with BCRABL gene amplification. Int J Cancer. 2008; 122(11):26212627.

19. Grosso S, Puissant A, Dufies M, Colosetti P, Jacquel A, Lebrigand K, Barbry P, Deckert M, Cassuto JP, Mari B and Auberger P. Gene expression profiling of imatinib and PD166326-resistant CML cell lines identifies Fyn as a gene associated with resistance to BCR-ABL inhibitors. Mol Cancer Ther. 2009; 8(7):1924-1933.

20. Beyazit Y, Kekilli M and Haznedaroglu IC. Secondgeneration BCR-ABL kinase inhibitors in CML. N Engl J Med. 2010; 363(17):1673-1675.

21. Bueno-da-Silva AE, Brumatti G, Russo FO, Green DR and Amarante-Mendes GP. Bcr-Abl-mediated resistance to apoptosis is independent of constant tyrosine-kinase activity. Cell Death Differ. 2003; 10(5):592-598.

22. Klejman A, Schreiner SJ, Nieborowska-Skorska M, Slupianek A, Wilson M, Smithgall TE and Skorski T. The Src family kinase Hck couples BCR/ABL to STAT5 activation in myeloid leukemia cells. EMBO J. 2002; 21(21):5766-5774.

23. Benekli M, Baer MR, Baumann H and Wetzler M. Signal transducer and activator of transcription proteins in leukemias. Blood. 2003; 101(8):2940-2954.

24. Hemmeryckx B, van Wijk A, Reichert A, Kaartinen V, de Jong R, Pattengale PK, Gonzalez-Gomez I, Groffen J and Heisterkamp N. Crkl enhances leukemogenesis in BCR/ ABL P190 transgenic mice. Cancer Res. 2001; 61(4):13981405.

25. Sattler M, Salgia R, Okuda K, Uemura N, Durstin MA, Pisick E, Xu G, Li JL, Prasad KV and Griffin JD. The proto-oncogene product $\mathrm{p} 120 \mathrm{CBL}$ and the adaptor proteins CRKL and c-CRK link c-ABL, p190BCR/ABL and $\mathrm{p} 210 \mathrm{BCR} / \mathrm{ABL}$ to the phosphatidylinositol-3' kinase pathway. Oncogene. 1996; 12(4):839-846.

26. Jacobberger JW, Sramkoski RM, Frisa PS, Ye PP, Gottlieb MA, Hedley DW, Shankey TV, Smith BL, Paniagua M and Goolsby CL. Immunoreactivity of Stat5 phosphorylated on tyrosine as a cell-based measure of Bcr/Abl kinase activity. Cytometry A. 2003; 54(2):75-88.

27. Kantrow SP and Piantadosi CA. Release of cytochrome c from liver mitochondria during permeability transition. Biochem Biophys Res Commun. 1997; 232(3):669-671.

28. Kuznetsov AV, Troppmair J, Sucher R, Hermann M, Saks V and Margreiter R. Mitochondrial subpopulations and heterogeneity revealed by confocal imaging: possible 
physiological role? Biochim Biophys Acta. 2006; 1757(56):686-691.

29. Lindsay J, Esposti MD, Gilmore AP. Bcl-2 proteins and mitochondria--specificity in membrane targeting for death. Biochim Biophys Acta. 2011; 1813(4):532-539

30. Altieri DC. Survivin and IAP proteins in cell-death mechanisms. Biochem J. 2010; 430(2):199-205.

31. Amarante-Mendes GP, McGahon AJ, Nishioka WK, Afar DE, Witte ON and Green DR. Bcl-2-independent Bcr-Abl-mediated resistance to apoptosis: protection is correlated with up regulation of Bcl-xL. Oncogene. 1998; 16(11):1383-1390.

32. Horita M, Andreu EJ, Benito A, Arbona C, Sanz C, Benet I, Prosper F and Fernandez-Luna JL. Blockade of the Bcr-Abl kinase activity induces apoptosis of chronic myelogenous leukemia cells by suppressing signal transducer and activator of transcription 5-dependent expression of Bcl-xL. J Exp Med. 2000; 191(6):977-984.

33. Nelson EA, Walker SR, Weisberg E, Bar-Natan M, Barrett R, Gashin LB, Terrell S, Klitgaard JL, Santo L, Addorio MR, Ebert BL, Griffin JD, Frank DA, et al. The STAT5 inhibitor pimozide decreases survival of chronic myelogenous leukemia cells resistant to kinase inhibitors. Blood. 2011; 117(12):3421-3429.

34. Warsch W, Kollmann K, Eckelhart E, Fajmann S, CernyReiterer S, Hölbl A, Gleixner KV, Dworzak M, Mayerhofer M, Hoermann G, Herrmann H, Sillaber C, Egger G, Valent P, Moriggl R, Sexl V, et al. High STAT5 levels mediate imatinib resistance and indicate disease progression in chronic myeloid leukemia. Blood. 2011; 117(12):34093420 .

35. Hoover RR, Gerlach MJ, Koh EY and Daley GQ. Cooperative and redundant effects of STAT5 and Ras signaling in BCR/ABL transformed hematopoietic cells. Oncogene. 2001; 20(41):5826-5835. 